\section{A larture}

\section{THERAPEUTIC INOCULATIONS OF BACTERIAL VACCINES.}

AND THEIR PRACTICAL EXPLOITATION IN THE TREATMENT OF DISEASE.

Delivered at the Medical Graduates' College and Polyclinic. Bx A. E. WRIGHT, M.D.DUBL., Eate Professor of Pathology, Army Medical Schocl, Netley; Pathologist
to St Mary's Hospital, W.

THE therapeutic method which I propose to consider with you to-day is a method as yet almost unexploited. None the less it is a method which is, if $I$ am not mistaken, destined to revolutionize our ordinary practice in dealing with localized bacterial invasions. In dealing with these our treatment has in the past consisted in making repeated applications of antiseptics or, in the case where this is impracticable, extirpating the seat of infection.

The time will come when, before embarking on either of these methods of treatment, and above all before acquiescing in a policy of leaving the bacterial invasion unchecked, an endeavour will be made in every case to arrest the invasion and to prevent its recurrence by calling into action the forces of resistance which lie latent in the organism. The physician of the future will, I foresee, take upon himself the rôle of an immunizator.

Defore developing my ideas in a more concrete form, and pointing out where the opportunities already lie for the exploitation of methods of immunization, it will be essential for as to obtain a clear conception of the immunizing reaction which is initiated by the inoculation of a vaccine. And we may group together under the appellation of vaccines attenuated living cultures of micro-organisms, sterilized cultures, and derivatives of such cultures.

It will be convenient, at the outset, to discriminate from the more complicated processes associated with actual disease the simpler reactions evoked by such vaccines. Let us note that in actual disease we have to deal with a reaction of immunity hampered and often frustrated by processes of necrosis and cell degeneration induced by the action of the bacterial toxins. In the case of properly-conducted vaccination procedures, we have to deal with processes of immunization uncomplicated and unfrustrated. It is with these immunizing reactions-with these, if I may so denote them, physiological reactions-that we have here to concern ourselves. In dealing with them I shall not even attempt to call up before you a mental picture of the hidden machinery in the protoplasm which elaborates the products of immunization which are found in the blood. You will already have been much wearied with such attempts. I shall content myself with setting forth to you the sequence of events which supervenes when the machinery of immunization is set in motion by the inocu. lation of a vaccine.

Sequence of Events after the Inoculation of a Bacterial Vaccine. The sequence of events after the inoculation of a bacterial poison was first clearly exhibited by Ehrlich1 in connexion with a series of inocula tions of tetanus toxin undertaken upon a milch goat already previously immunized. The curve of immunity (Fig. x) here reproduced from immunized. The curve of immunity
CURVE

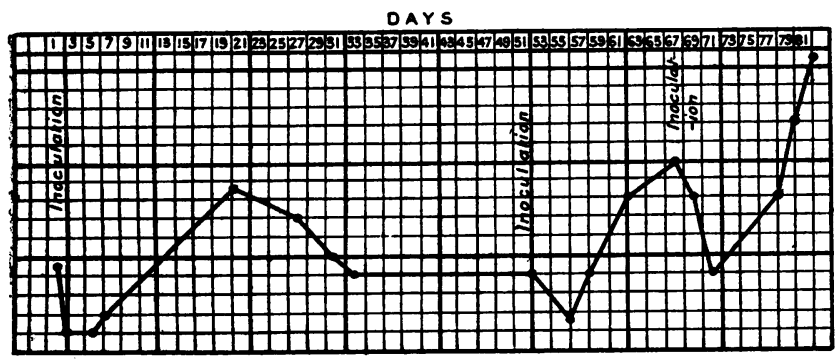

Fig. r.-Curve of the immunization reaction as obtained by measuring the contents of tetanus antitoxin

\section{quent to three inoculations of tetanus toxin.}

Ehrlich and Brieger's paper, discloses the content of the milk in tetanus antitoxin at different intervals after the inoculation. The following are the points to be noted:

x. Immediately subsequent to the inoculation of tetanus toxin we have 4 what we may conveniently speak of as a " negative phase" of the curve of immunity.

2. This negative phase is succeeded after an interval by a "positive phase." In the case of the first inoculation here in question the climax of the positive phase was marked by a duplication of the previous anti toxic power of the milk.

3. After a further interval the curve comes back to the higher base line which represents the quasi-permanent achievement of the immunization process.

The same succession of a negative and a positive phase is reproduced after the second, and, so far as the curve was traced, after the third inoculation.

The reaction of immunity was next studied by Salomonsen and Madsen in connexion with inoculations of diphtheria toxin undertaken upon a previously immunized milch mare. A reference to the curves in Fig. 2 (below) which represent the antitoxin-content in the milk and blood respectively, will show that we have here again to deal with a negative and positive phase.

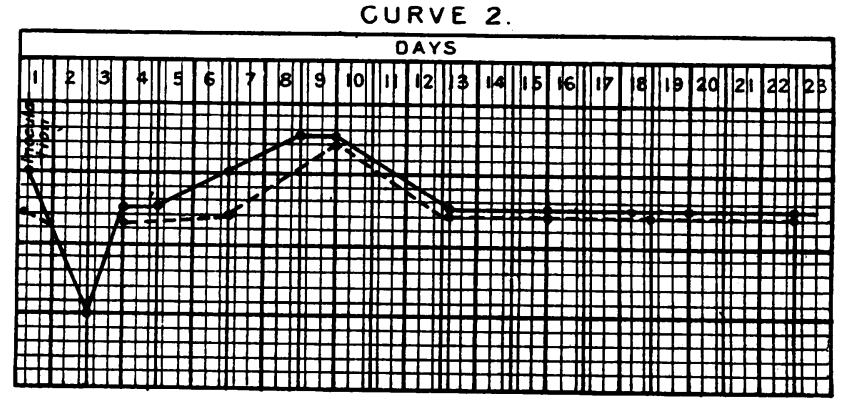

Fig. 2.-Curve of the immunization reaction obtained by measuring the content of the blood and milk of a mare in diphtheria antitoxin after the inoculation of diphtheria toxin. Unbroken line -, antitoxin of milk; dotted line......, antitoxin of blood.

Similar curves have been obtained in connexion with other immunization processes, in particular by Bulloch ${ }^{3}$ in connexion with the inoculation of ox-blood into rabbits, and by Morgenroth in connexion wi th the inocuiation of rennet.

I myself have, in a series of observationst made on men, obtained evidences of a negative and a positive phase of the bactericidal power of the blood after typhoid inoculation. Two curves showing a negative and positive phase of bactericidal power after typhoid inoculation are presented in Fig. 3 (p. ro7o). In later experiments undertaken by the same method I have obtained evidence of the maintenance of a higher base line two years after inoculation.

In a further research, dealing with the effects of antistaphylococcus noculations 6 upon a series of men evidence was obtained by me of the supervention of a negative and a positive phase of phagocytic ${ }^{7}$ power upon the inoculation of staphylococcus vaccines. I showed that in association with the decline and rise of the phagocytic power, an aggravation and improvement manifested itself in the clinical symptoms. A typical curve is set forth in Fig. 4 (p. ro70).

I think it may be taken as definitely established by the above that we have to deal in connexion with every immunization process with a succession of a negative and positive phase. So far we have concerned ourselves only with a typical form of curve. We should, however, lapse into fallacy if, carrying away in the mind's eje the features and general proportions of such typical curves as are shown in the figures above we w ere to assume that these features and proportions must be:lreproduced in the case of every inoculation process. And similarly we should fall into error if we were to assume that a series of successive inoculations would in every case, as in Fig. $r$, lead to the achievement of a high base line of immunity.

We shall best guard ourselves against errors such as those first referred to if we consider the curves relating to the effect of antityphoid inoculation set forth in Figs. 5 and 6 (p. 107o). In curve 5 the negative phase is suppressed, or speaking more strictly, the positive phase is found fully developed twenty-four hours after inoculation. In Curve 6 the nadir of the negative phase is not reached till the ninth day after the inoculation the frrst evidence of 2 return in the direction of a positive phase being obtained on the fifteenth day.

Let it be noted here that the differences in the duration of the negative phase set forth in these traces are due either to comparatively small differences in the dose of vaccine administered or to, idiosyncrasies on the part of the patients. If I may judge from experience of the results of the self-inoculation of a much larger dose of typhoid vaccine and from the results of blood examinations undertaken upon patients convalescent after very severe attacks of typhoid fever, it would seem to me that the incorporation or, as the case may be, generation in the system of e xcessive quantities of typhoid poisons may be followed by a negative phase of very many months' duration.

What applies in the case of single inoculations applies with even greater force to the case where a series of successive inoculations is undertaken It is of fundamental inportance to recognize that the effect of every such series of inoćulations is a cumulative one. It is cumulative in the sense of the negative phase and the poisoning of the organism when the doses of vaccine are excessive, and when they fall upon the negative phases of 

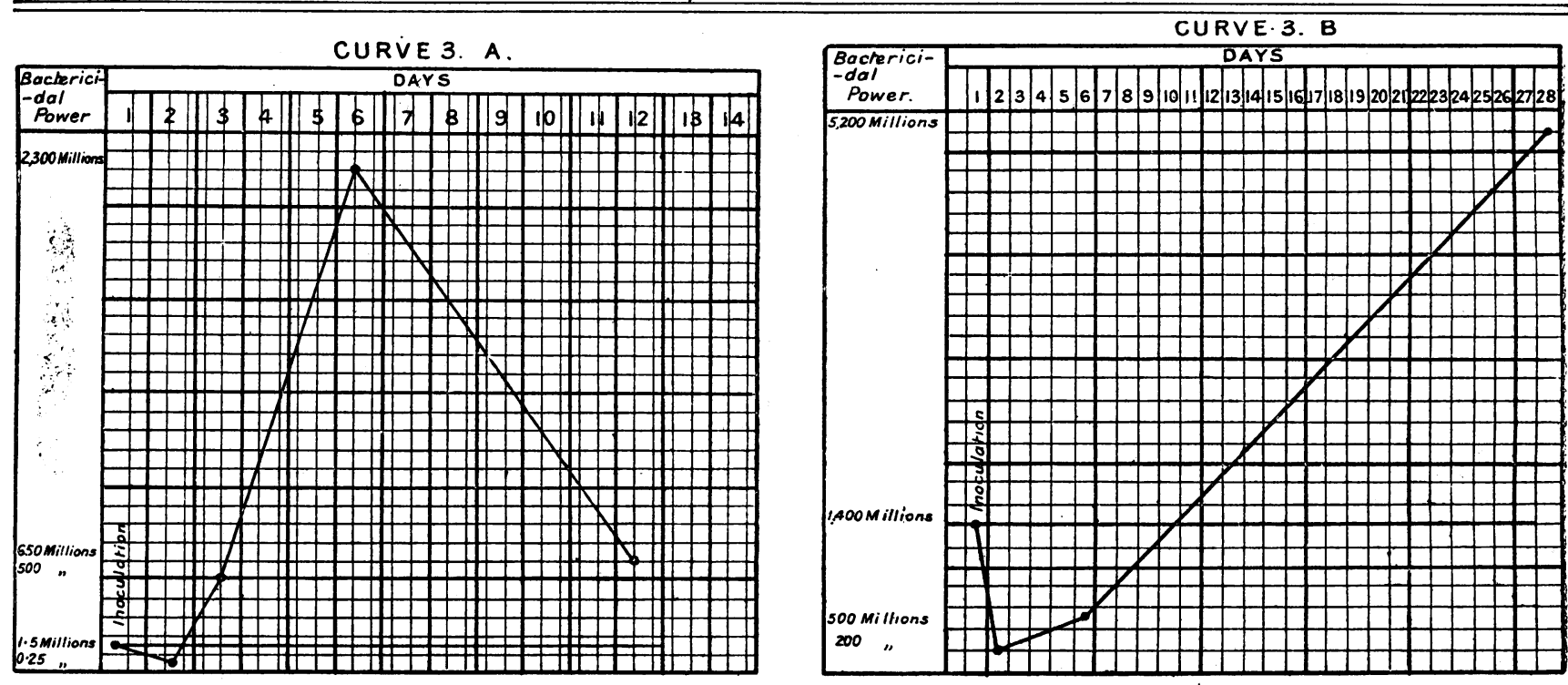

Flg. 3.-Curves (A and B) of the immunization reaction obtained by measuring the bactericidal power of blood obtained from the finger before and after the inoculation of antityphoid vaccine. In Curve A all the measurements on the upper.(positive) side of the base line have for convenience of exhibition been represented on a hundred-fold reduced scale.

CURVE 4 .

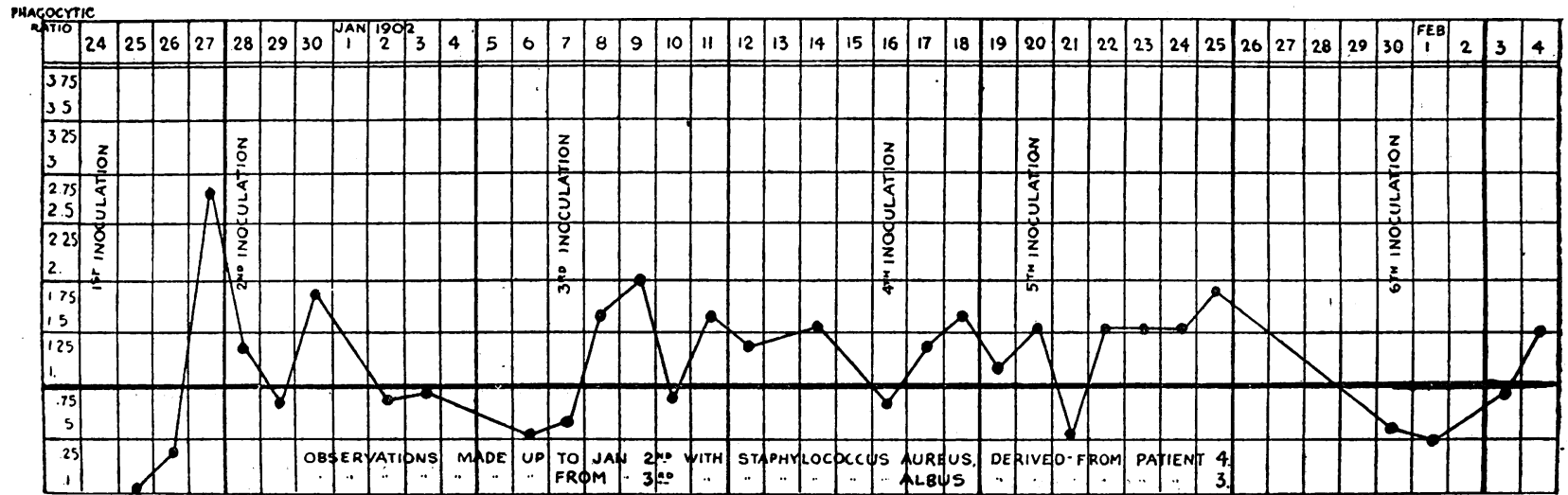

Fig. 4.--Curve of the immunizacion reaction produced by a series of successive inoculations of a staphylococcus vaccine; the curve represents the phagocytic ratio of the patient's blood, the phagocytic power of the control blood being everywhere taken as $\mathrm{I}$. the foregoing inoculations. And only in the case where the doses are properly adjusted; and where each succeedinginoculation starts from a higher level attained by the previous inoculation will the effect of a series of inoculations be cumulative in the direction of the positive phase and in the direction of raising the organism to a higher level of immunity.

$$
\text { CURVE } 5 .
$$

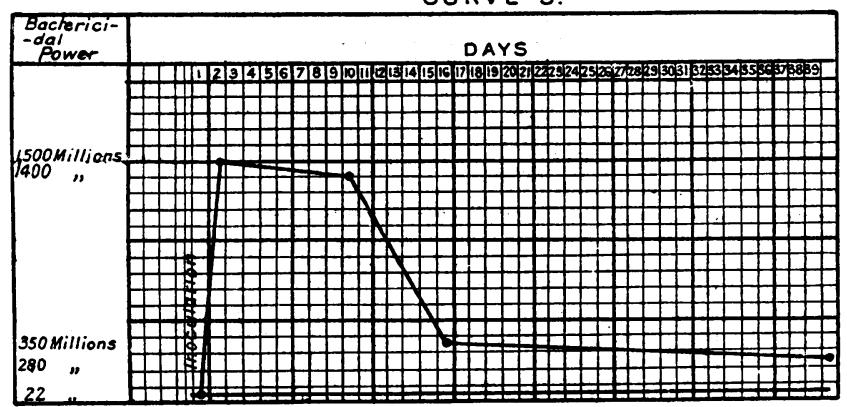

Fig. 5.-Curve'of the immunization reaction obtained by the inoculation of antityphoid vaccine, suppression of the negative phase.

You have now before you in outline what is known with regard to the sequence of events following upon the introduction of a vaccine into the organism, and with regard to what I may call the law of the negative land positive phase and of the attainment of the higher base line. I desire to insist upon the fundamental practical importance of this law in connexion with every immunization procedure. Let me, with a view to illustrate its practical' importance, deal, before I take up the subject matter proper of $\mathrm{my}$ diseourse, very briefly with the negative and positive phase in connexion with prophylactic inoculations of bacterial cultures, and in connexion with serumtherapy.

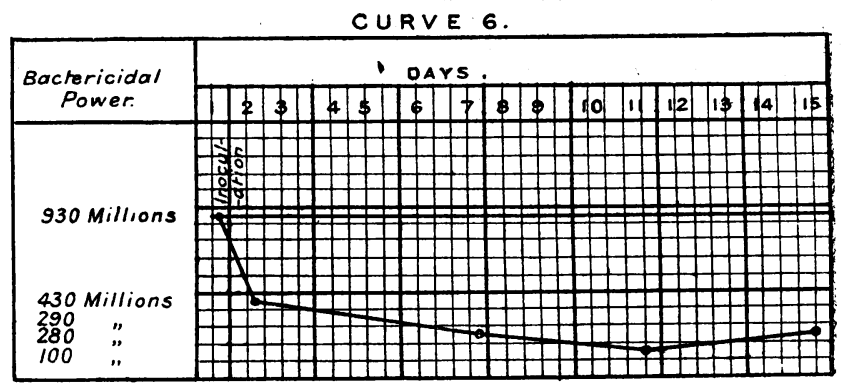

Fig. 6.-Curve of the immunization 'reaction 'obtained by the inoculation of antityphoid vaccine, postponement of positive phase.

Practical Importance of the Law of the Negative and Positive Phase in Connexion with Prophylictic Inoculations Undertaken with Living or Sterilized Vaccines.

In considering, in the light of individual results obtained in certain series of antityphoid inoculations undertaken in actually infected surroundings, the significance of the negative phase of bactericidal powerafter antityphoid inoculation, I have called attention to the fact that the success of these prophylactic inoculations is imperilled where excessive doses of vaccine are administered to patients in actually infected surroundings, or immediately before transierence to suck surroundings. 
Basing myself upon information collected in India by the Indian Plague Commission, ${ }^{8}$ in connexion with Mr. Haffkine's antiplague vaccination, I made a similar suggestion in connexion with this prophylactic inooulation. And I have recently learned that, the idea of a risk attaching to the inoculation of large doses of vaccine in infected surroundings suggested itself also to several observers in South Africa who had occasion to watch the effect of the antiplague inoculations there undertaken If the suggestions made by $m e$ in connexion with antityphoid and antiplague inoculation are justified, we may not unreasonably expect to find indications of an increased susceptibility to small-pox in the period supervening immediately upon the development of vaccinia pocks. It seems to me that such evidence can be foun $q$. In this connexion attention may be drawn to the fact that a vaccine lymph which has altogether failed to develop may, in the case where a more potent vaccine lymph is afterwards successfully inoculated, take on a typical development. We can hardly explain this fact otherwise than by assuming that the resisting power of the patient which sufficed to hold in check the earlier applied and.weaker vaccine is diminished or abolished under the influence of the toxins elaborated in the course of the development of the subsequently inoculated and more active vaccine.

Evidence of similar import, but from the nature of the case less conclusive evidence, is supplied by certain of the records of small-pox attacks, and in particular by records of fatal small-pox attacks affecting those who had been vaccinated immediately before. I would point out that it is traditional in these cases to ascribe the small-pox attacks in question to infection contracted previous to vaccination. It is also traditional to vindicate this ascription by reference to the longer incubation of small-pox, overlooking the possibility that the incubation period may be shortened where the resistance of the organism is reduced.

$A$ final word on this question will be appropriate. If the risks incidental to the production of a negative phase attach, as; I believe they do, to prophylactic inoculations in the case of all septicaemic diseases alike, it is obviously incumbent upon us neither to ignore nor to magnify these dangers, and, above all, to recognize that these risks can be minimized. Let it be observed that the risk of a negative phase comes seriously into consideration only when excessive doses of vaccine are employed and when the prophylactic inoculations are undertaken in the actual presence of infection. The remedy lies at hand. It lies in the case where a sterilized bacterial culture is employed in the reduction of the dose. It lies in the case of anti-small-pox vaccination in the reduction of the number of insertions; in other words, in limiting the elaboration of toxins by diminishing the area of skin surface employed for the culture of the organism of vaccinia.

Practical Importance of the Appreciation of the Iaw af the Negative and Positive Phase in Connexion with the Therapeutic Inoculation of Sera derived from Animals vicariously Inoculated with Bacterial Vaccines.

The principle of serumtherapy-that is, the idea of transferring to patients already the subjects of bacterial infection immunizing substances withdrawn from animals vicariously inoculated-appeals in a very forcible way to the medical mind by the fact that it promises a rational treatment of all bacterial diseases, and by the fact that it has fulfilled that promise in the case of diphtheria.

The prestige which it has derived from this signal success has led to the exploitation of the method of serumtherapy in connexion with almost every bacterial disease, and the time would appear to have arrived for a general survey of the results. We can hardly fail to recognize that the new method of treatment has almost everywhere (I except, of course, the case of diphtheria) failed to do appreciable good. I would also have you note that positive harm may upon occasion result from the resort to a serum. I may enforce this last statement by practical examples.

(r) There was submitted to the Indian Plague Commission a particular " antiplague serum" intended for therapeutic administration. This serum, which was tested by me as a member of the Commission, precipitated the death of every plague-1nfected animal to which it was administered, the acceleration of the fatal issue being in each case directly proportional to the dose of serum administered. ${ }^{9}$ (2) In examining at Netley a number of " antityphoid" sera with a view to selecting for employment in South Africa any one that might seem calculated to be therapeutically useful, I found a particular " antityphoid serum" which completely abolished the bactericidal power which normal human serum exerts upon the bacillus typhosus. (3) I have myself administered with therapeutic intent to two Malta fever patients (personal friends) an " anti-Malta-fever serum," which produced unmistakeably toxic effects. The serum in question was a serum of my own preparation. (4) My friend Dr. Bulloch has had occasion to test on guinea-pigs the effect of an "antistaphylococcus serum." The serum in question was found to exert a lethal effact in doses of $x$ c.cm. and over. (5) The same has been observed by Marmorek in connexion with certain antistreptococcus sera, and instances are, I think, not infrequent-they have certainly occurred within my experience-in which the administration of "antistreptococcus serum" has seemed to produce toxic effects. (6) A number of different sclentific workers have, in endeavouring to prepare an "antituberculous serum" by the inoculation of tuberculin into horses, ob- tained a serum which produced on inoculation into man and animals an unmistakeable tuberculin reaction. (7) Lastly, Dunbar 10 records the development of toxic effects in the case of a patient inoculated with an "anti-hay fever." serum.

These facts constitute, I think, striking confutation of the idea commonly entertained in our profession that all that is required for the production of an effective therapeutic serum is the incorporation into an animal of progressively increasing doses of a bacterial culture, and the withdrawal of the serum ten days after the last injection. In other words, the facts above enumerated enable us to realize that in default of an active production of immunizing substances on the part of the animal vicariously inocalated, the sera which are drawn off will inevitably possess the toxic properties of vaccines originally inoculated. Our awakening to this fact must be signalized by a re-examination of the whole question of serumtherapeutics. That examination must be undertaken in the light of the facts which have been adduced above with regard to the negative and positive phase.

Viewed from this point of view, the interpretation of "the untoward results which followed the administration of the "antiplague serum" reierred to above presents no difficulty. In point of fact, an inspection of the horses from which the serum in question had been derived showed that the horses were still suffering from the fever and local disturbance caused by the inoculation of the plague toxins. The serum had, in other words, been drawn off during the negative phase.11

Let it, however, be carefully noted that the passing off of the local and constitutional symptoms does not in itself afford any guarantee that the negative phase has given place to a positive phase. Personal experience in connexion with the inoculation of typhoid and Malta fever cultures inte horses, the similar experience of others in connexion with the inoculation of other poisons into animals; and, lastly, experience of the effect exerted on human blood by antityphoid inoculation and by typhoid fever itself make it certain that the persistence of the negative phase does not always betray itself by physical symptoms.

There is warranty for going further; there is, in fact, warranty for asserting that the persistence of the negative phase would in many cases be undetected by our present methods of testing sera.

Thus, for instance, the verification of the presence of agglutinins in the serum which has been withdrawn does not in any way guarantee the neutralization of the toxic substances which have been inoculated into the animal. Let it be observed in this connexion that the toxic "antityphoid" and "anti-Malta fever" sera referred to abova pos. sessed high-in the former; indeed, exceptionally high-agglutinating powers.

Again experiments undertaken upon laboratory animals afford no secirity for the absence of toxins from the sera under examination, unless the animals are equally sensible with man to the particular toxing which are in question.

These points with regard to the difficulty of eliminating from nse negative phase) sera are, it will be seen, of altogether general applic :tion. If they have been overlooked, this has no doubt been owing to the fact that it has been possible, in the case of the serumtherapeutics of diphtheria, owing to the sensibility of the'guinea-pig to diphtheria toxin. to eliminate all toxic (negative phase) sera from use, and thus evary thing has in this respect here gone well.

The elimination of sera drawn off in the negative phase is, as con. sideration will show, only one of the preconditions of a successful ser untherapy. A further precondition of the successful exploitation of an antitoxic serum is that there shall be transferred to the patient in the few cubic centimetres of foreign blood which it is possible to administer a sufficiency of antitoxin to neutralize at least a sensible fraction of all the toxin which is being elaborated in the patient's system. In other words, it is essential that the antmal which is selected for vicarious inoculation shall possess an al together phenomenal power of reaction with respect to the particular bacterial poison employed. This condition, though it is satisfled in the case where horses are vicariously inoculated with diphtheria and tetanus toxins, is not, so far as is at present known, satisfiod in the case where animals are inoculated with any other poison.

superadded to the difficulties already adverted to are other and greater difficulties which have reference to the therapeutic exploitation of antibacterial sera. I refer to the difficulties created, on the one hand, by the circumstance that bactericidal elements disappear from the serum very soon after the blood has been withdrawn, and on the other hand by the circumstance that these bactericidal elements may be incapable of developing their effect when transferred from the vicariously inoculated animal to the human patient.

Let me finally, before taking up the subject matter proper of my discourse, try to sum up for you the situation. The path of the immunizator who desires to proceed by the method of serumtherapy is bestrewn with all manner of formidable difficulties. He must, in the first place, find methods which shall enable him to exclude: from use sera drawn off during the negative phase. He must, in the second place, achieve the hyperimmunization of the animal vicariously inosulated. Lastly, in the case of antibacterial sera, he must find means of preserving the products of immunization unaltered after withdrawal, and of securing that they shall be operative within the system of his patient. 
Therapeutic Inoculations of Bacterial Vaccines Undertaken upon Patients Already the Subjects of Bacterial Invasion.

If the situation as regards serumtherapy is as I have set it forth to you it manifestly behoves us to cast about and see whether there may not be a more excellent way. I venture to submit to your consideration-and here I arrive at last at the subject matter proper of this discourse-the suggestion that that more excellent way may, perhaps, in many cases be found in the therapeutic inoculation of the patient with a bacterial vaccine. Let me anticipate two a priori objections.

Let me make it plain, in the first place, that $I$ am very far from suggesting the incorporation of additional bacterial toxins into a patient already the subject of a septicaemic disease or a serious bacterial intoxication. Such an inoculation could, I take it, serve no good purpose, inasmuch as the bacterial elements similar to those that constitute the vaccine would in such a case already be circulating in the blood stream. Furthermore, as you will immediately appreciate, the superaddition of additional bacterial toxins would inevitably prolong or reproduce the negative phase, if, indeed, it did not definitely turn the scale against the patient.

The suggestion I make is that bacterial vaccines should be employed in the case where we have to deal with localized bacterial invasions associated with inflammation at the site of inoculation. The situation is here entirely different from that which has to be confronted in septicaemic diseases. On the one hand, the conditions are here already unfavourable to the invasion of the blood stream by micro-organisms. and there is, on the other hand, a considerable uncalled-on reserve of resistance on the part of the organism. We have to deal, in fact, with a situation not altogether unlike that which obtains in the case of already partially immunized animals, such as those employed by Ehrlich and Salomonsen in the experiments which we considered at the outset of this lecture. Holding as we do in such a case a considerable balance in hand, we are in a position to confront without alarm the prospect of a possible temporary diminution of that balance. We are, in fact, in a position to adventure something for the sake of achieving afterwards a positive phase of increased resistance.

A further a priori objection which must be reckoned with is the followIng : I may throw it into the form of a question. If it was legitimately argued above that an inoculation of a bacterial vaccine would serve no good purpose in a case where the bacterial elements which call forth the immunizing reaction are already circulating in the blood, does not this argument apply also to the case where bacterial toxins are absorbed into the system from a localized seat of infection? In point of fact it seems not to apply. The explanation which suggests itself is that in such cases only the products of the metabolism of the bacteria, as distinguished from the substances in the bacterial protoplasm which evoke a production of antibacterial substances, are absorbed into the blood stream.

What I picture to myself as the situation will perhaps appear to you in a clearer light if I place before you the following series of facts :

The blood of the guinea-pig-and for our purposes we have to consider only the male cavy-contains no spermatoxin; in other words, it exerts no poisonous effect upon the cavian spermatozoa. This is in conformity with the circumstance that, while, conceivably, certain other metabolic products are absorbed into the blood from the testicle, the spermatozoa themselves and their constituent elements are normally not so absorbed. The conditions are here presumably analogous to those which obtain in the case where bacteria are cultivating themselves locally in the organism.

If we now, after extirpating one of the guinea-pig's testicles, inoculate him subcutaneously with a suspension of spermatozoa obtained by making an extract of the extirpated testicle, we find that his blood develops spermatoxic properties, and that when brought into contact with activgly moving spermatozoa it immediately arrests their movements. Without pressing home the analogy, we might on a similar principle expect to induce an elaboration of antibacterial substances by inoculating a bacterial culture into a patient already the subject of a localized infection.

Having, so far as I can, disposed of the a priori objections which might suggest themselves in connexion with the proposed method of therapeutic inoculation of bacterial vaccines, and having given you, I hope, a certain insight into the principles of the proposed method, I will now ask you to consider where the opportunities lie for the application of the method. I may appropriately begin by discussing those therapeutic inoculations of which I have already had some experience.

Treatment of Furunculosis, Sycosis, Acne, and Localized Staphylococcus Infections Generally by the Inoculation of a Staphylococcus Vaccine.

There can, I think, be no doubt that in acne vulgaris, furanculosis, and most cases of sycosis we have nearly always to deal with an invasion of the skin follicles, or, as the case may be, of the subcutaneous tissue by the staphylococcus pyogenes. In almost every case the suppurating areas yield the staphylococcus in pure culture. This, however, is only one of the factors in the causation of these disorders.

Another factor is revealed by an investigation of the phagocytic power of the blood. This examination has, in all the cases I have as yet dealt with, revealed a defective power of phagocytosis with respect to the staphylococcus. We have, it seems to me in the above, taken in connexion with the extreme chronicity, tendency to relapse, and comparative non-infectivity which characterizes these disorders, clear indications of the line of treatment that ought to be adopted. We ought to aim primarily at the immunization of the patient against the staphylococcus.

I have elsewhere 12 given an account of six consecutive cases which were treated by the inoculation of a staphylococcus vaccine, and have set forth the details of the blood examinations, and in association with them the clinical result. In two of these cases-marked, but only temporary, improvement was achieved; in the four others-acomplete and very rapid cure was effected. The cases in which the improvement was only partial and temporary were-a case of boils and irritable pimples occurring in an elderly lady as a sequel of a severe operation, and $-a$ case of extensive sycosis and eczema barbae dating back eight years to almost the period of puberty. In both of these cases there appears to have been a deficient power of response to the inoculations.

Among the cases cured, the most conclusive from the point of the previous chronicity of the morbid process, the severity of the symptoms, and the subsequent following up of the case, was that of an officer who, subsequent to a septic infection which eventuated in peritonitis, had for a period of seven years been a victim to sycosis, eczema barbae and tarsistyes in the eye, and deep and superficial furuncles. All these troubles. disappeared after three successive inoculations of staphylococcus vaccine. His face had remained absolutely free from eruption when the patient, eighteen months after the date of his original first inoculation, presented himself for reinoculation on account of the development of two incipient boils, these being the first which had developed since undergoing the treatment. The reinoculation was effectual in checking any further relapse.

It may be added that, owing to the relatively small toxicity of staphylococcus cultures, the therapentic treatment by staphylococcus vaccine involves comparatively little discomfort. The discomfort is, at any rate, absolutely trivial in comparison with the mental suffering associated with disfigurement due to acne or sycosis, and the physical evils associated with the reiterated application of antiseptics, epilation, and the fomentation and lancing of furuncles.

If staphylococcus vaccine should continue to approve itself aseiul in the treatment of localized cutaneous invasions of the skin, this would manifestly pave the way for further therapeutical applications in connexion with staphylococcus invasions of wounds and open ulcers and granulating surfaces generally.

Treatment of Cholelithiasis, Appendicitis, Colitis (also of Cystitis, Pyelitis, and Endometritis where these are Produced by the Colon Bacillus) by the Therapeutic Inoculation of a Coli Vaccine.

The colon bacillus, even if we consider it quite apart frow its near congeners, the dysentery bacillus, the Gaertner bacillus, and the typhoid bacillus, all of which, like the colon bacillus, affect by predilection the lower portion of the ileum and upper end of the large intestine, is responsible for an altogether astonishing amount of human ills. The rôle which the colon bacillus plays in connexion with internal surfaces is, in fact, "not less predominant than that played by the staphylococcus in connexion with external surfaces and wounds. And the gravity of the local inflamma tory processes which result from the inroads of the colon bacillus is infinitely greater. These derive their gravity, first, from the importance of the organs which are involved, secondly, from the anatomical relations of these organs with the peritoneum, thirdly, from the practical impossibility of effectively applying antiseptics, and, fourthly, from the very serious surgical procedures which are required in the case where the localized invasion culminates in the production of pus and shows a tendency to generalize itself. All these are considerations which plead in favour of dealing with colon infections by the method of immunization.

Time will not permit of my referring to more than one or two of the many problems which open out before us when we take into consideration the possibility of dealing with colon bacillus infections or by the method just suggested. And yow will understand that even if time did permit, the limitations of my knowledge in this imperfectly explored field would very soon impose a restraint upon me. It may, however, not be altogether unprofitable to suggest to you in connexion with this question certain lines of thought.

Cholelithiasis.-Evidence amounting now almost to demonstrative proof has been accumulating showing that the formation of gall stones is dependent upon bacterial invasion of the gall bladder, just in the same way as the formation of the ordinary phosphatic calculus in the bladder is dependent upon a bacterial invasion. It has further been established that the colon bacillus is the particular micro-arganism 
which is responsible for the formation of gall stones. Not only is this micro-organism found in pure culture in the gall bladder in most cases of cholelithiasis, but it is found also in the interior of practically all recent biliary concretions. Furthermore, it has been shown that gall stones can be experimentally produced by a direct inoculation of the colon bacillus into the gall bladder. And, lastly, it is interesting and important to note that Professor Vaughan Harley has recently found that ordinary gall stones are spontaneously dissolved when they are introduced into the gall bladders of dogs, while no such solution occurs when they are introduced in association with pus derived from the inflamed gall bladder of man.

It would seem, in view of the above, that the treatment of cholelithiasis ought to be directed to the immunization of the patient against the colon bacillus. We can see that it is within the bounds of possibility that the colon bacillus infection might in this way be checked, and that the gall stones might be dissolved. Even if these ends were not achieved the immunization would at least prepare the way for surgical procedures by diminishing the attendant risks. Let us note with regard to the efficacy of these surgical procedures that the removal of the gall stones would not by itself make an end to the evil.

Appendicitis.-More important by far than the pathology of gall stones is the pathology of appendicitis. Without for a moment contending that the colon bacillas is the only microorganism involved in the etiology of appendicitis, it appears to me to be beyond doubt the essentially important agent. I find, for instance, on looking up my records of bacteriological examinations undertaken in the surgical theatre or, as the case may be, the post-mortem room at St. Mary's, that the colon bacillus was in eight successive cases obtained in pare ealtivation from the pus, and that it was obtained in pure culture from the contents of the caecum or appendix in 5 out of the 6 cases in which cultures were made from the contents of the intestinal canal.

Here again it would seem to me that treatment ought to be directed to the immunization of the patient against the colon bacillus. Immunization procedures might, it seems to me, appropriately be undertaken on the one hand with the design of preventing a recurrence and avoiding operation, and on the other hand with the design of preparing the patient for operation in those cases where surgical procedures are postponed until after the subsidence of the symptoms of inflammation. The risks of inducing peritonitis which are incident to the necessary breaking down of adhesions and opening up of old foci of infection might conceivably be appreciably diminished by these means. The results which have been obtained in man by anticholera and antityphoid inoculations, and animals by anticoli inoculations, would seem to show the possibility of effective immunization against bacterial infections proceeding from the intestinal canal.

Cystitis and Localized Bacterial Infections of the Gexitourinary Tract.

It is hardly necessary to do more than advert to the frequent association of cystitis and pyelitis with an invasion of the urinary tract by the colon bacillus. It is perhaps less well known that certain cases of endometritis are associated with an invasion of the uterus by the same microorganism. It would seem possible that patients, the subjects of these infections, might be advantageously treated by inoculations of coli vaccine. In two cases in which I have already carried out such inoculations, the patients have claimed that they derived benefit from them. I was, however, unable to detect any objective evidence of improvement by bacteriological examination of the urine. It is obvious, however, that the number of coli bacilli in the urine is not necessarily a correct index of the condition of the local inflammation process in the wall of the urinary tract.

\section{Treatment of Tuberculosis by Therapeutic Inoculations of} Tubercle Vaccine.

A few words by way of introduction to the discussion of this question may perhaps serve to place matters clearly before you. The treatment of tuberculosis by tuberculin as originally introduced by Koch was in intention a method of toxitherapeutics. The tuberculous toxins which were inoculated were intended to compass the expulsion and destruction of the invading tubercle bacilli by effecting necrotic and degenerative changes in the foci of infection. It is now universally recognized that this therapeutic principle was fundamentally erroneous. It is unnecessary to say more, except that the results were untoward and that the necrotic, degenerative, and inflammatory changes which were effected in the foci of infection contributed, in some cases, to the disseminstion of the tubercle bacilli in the infected system.

Of recent years modifications have been made by Koch in the preparation of the tuberculin which have as their result the elimination of the more soluble toxins and the incorporation. into the menstruum of the less soluble elements of the bacterial protoplasm. In short, modifications have been made which bring tuberculin (the so-called new tuberculin or $O$ and $\mathbf{R}$ tuberculins) more into line with our ordinary bacterial vaccines. Following upon these changes a complete change of policy has been tacitly inaugurated. The tuberculin inoculations have been definitely divested of the character of toxitherapeutic inoculations, and have been definitely invested with the character of therapeutic inoculations of a tubercle vaccine designed to call forth an antibacterial reaction in the organism.

As soon as we consider the antitubercle inoculations from this standpoint, everything that has occurred in connexion with them becomes immediately intelligible. We. appreciate, in the first place, that the untoward results which so often supervened upon the inoculation of Koch's. original tuberculin were, in 80 far as they were not the: result of the degenerative and inflammatory changes above referred to, imputable to the inappropriate character of the vaccine, and often, no doubt, to the induction of a cumulative negative phase under the influence of successive inoculations uncontrolled by intermediate blood examinations. We appreciate further that the outlook for a useful exploitation of therapeutic inoculations for tubercle is more hopeful now that we have at disposal a vaccine which contains in it elements derived from the bacterial protoplasm ; and now that we have at disposal in the tuberculous serumsedimentation reaction a method by which we can obtain some information of the progress of the reaction of immunization in the patient's syatem. I would submit to you that since we now need no longer work entirely in the dark, we ought cautiously to exploit in the treatment of localized tuberculous affections the tubercle vaccine which we owe to the ever fertile. labours of Koch.

Treatment of Bacterial Infections of the Meninges, of the Membranes of the Respiratory Tract, of the Middle Ear. of the Uterus, and of Joints by Therapeutic Inoculations of the Appropriate Vaccines.

Before proceeding to summarize the general results we have arrived at, I may perhaps take an opportunity of explaining that the therapeutic method which is here in question is capable of an even more extended application than that sketched out above. I foresee that if the principle of the therapeutic inoculation of bacterial vaccines establishes itself, as I do not doubt that it will in the future, as an approved method for the treatment of chronic and recurrent inflammation procerses, it will be exploited also in connexion with chronic and recurrent meningeal disease ( $I$ have in view here in particular certain cases of infantile paralysis), middle-ear disease, and chronic bronchial uterine catarrh, and chronic joint affections. Every such case would, of course, be submitted to bacteriological examination with a view to the determination of the determining cause of the infection, and the employment of the appropriate bacterial vaccine. In considering such a treatment in connexion with a meningeal infection there may be borne in mind the fact that we have, in the case of the antirabies inoculations, a demonstration of the possibility of checking, by the means of subcutaneous therapeutic inoculation of a vaccine, the spread of infective micro-organisms in the cerebro-spinal system. Similarly, in considering a possible application of bacterial vaccine in connexion with inflammation processes affecting the mucous membranes of the respiratory system, we may derive encouragement from the results obtained by Professor Dunbar in connexion with inoculation against hay fever. For an elaboration of an antitoxin against the pollen toxin in the organism of animals has been achieved by him and he has obtained indications. of a similar production of antitoxin in the organism of: a susceptible human patient.

\section{SUMMARY.}

Let me, finally, recapitulate to you the conclusions we have arrived at in the course of our study :

1. We have seen that we have in connexion with every immunization process a sequence of negative and positive phase 
followed in the case where the inoculation is successful by the maintenance of a higher base line of immunity.

2. We have seen that the inoculation of an excessive dose may involve a risk, in particular, the risk of an undue prolongation of the negative phase.

3. We have seen that the inoculation of a series of doses of a vaccine will, in the case where the inoculations are uncontrolled by intermediate blood examinations, involve the possibility of the production of a cumulative negative phase.

4. We have seen that the cumulative positive phase which is a desideratum either in itself or as leading to the maintenance of a high base line of resistance, is achieved only when the successive doses are properly adjusted and when the inoculations are appropriately interspaced.

5. We have seen that the success of a prophylactic inoculation process may be imperilled where sequence of negative and positive phase, and the cumulative effect of successive inoculations, is not taken into consideration.

6. We have seen that the success of serumtherapy in diphtheria and its comparative failure in the case of other diseases is explained by the fact that in the first case we are able to secure the elimination of all negative phase blood, and we are able to induce in the vicariously inoculated animals a cumulative positive phase of absolutely phenomenal dimensions. In the case of other diseases we have been unable to secure these prerequisites of a successfal serumtherapy.

7. We have seen that in the case of patients who, though suffering from localized bacterial invasions, are possessed of a considerable balance of resisting power, it is possible without risk to undertake therapeutic inoculations of bacterial vaccines, provided always that the results of these inoculations are controlled by subsequent blood examinations.

Let us realize, in conclusion, tha $i$ when all has been done that can be done in the way of guarding a patient against the risks attaching to the negative phase the success of a therapeutic inoculation cannot be guaranteed. The success must in each case depend upon the power of response which is possessed by the indivi dual. In the case of a particular patient that power of response may fail us. It may fail us also in connexion with particular bacterial infections. But it may be predicted that success will in some cases be achieved. It geems to me, for instance, that it will certainly be achieved in the case of simple staphylococcus infections occurring in the young and otherwise robust. It seems to me very probable that it will be achieved also in the case of certain coli infections.

REFERENCES.

1 Zeit.f. Hyg., vol. xiii. 2 Ann. de l'Inst. Pasteur, 1887. 3 Trans. Path. Soc. s902. 4 Lancet, September r4th, rgor. SThe method employed was that described by me in the Proc. Roy. Soc., vol. Ixxi. ${ }^{6}$ Lancet, March 29 th, 1902. 7 The method employed was that described by Leishman, BRITISH MEDI CAI JOURNAL, January Irth, rgo2. 8 Report, Indian Plague Commission, - Report, Indian Plague Commission, chapter v. 10 Dunbar, Ursache $u$. specifische Heilung des Heufiebers, 1903. 11 Report, Indian Plague Commission, loc. cit. ${ }^{12}$ Lancet, March 26th, 1902.

\section{ON EXALTATION OF BACTERIAL VIRULENCE BY PASSAGE IN VITRO.}

By E. A. SHAW, M.B.CantaB.,

THE appearance of Dr. E. W. Ainley Walker's paper on this subject in the British Medical Journal of October I8th, 1902 , leads me to place on record the following series of observations made at Netley during the spring and summer of I90I, and not hitherto published because it was hoped that later more unimpeachable evidence of increased virulence by this method would be obtainable, and that the series might be extended by the inclusion of micrococcus Melitensis. Illness, the exigencies of the service, and other causes have conspired to prevent the realization of these desiderata, which, however, it is hoped may be attained ere long

The medium selected for the "passages" was fresh human blood serum obtained from myself; the methods of dilution of cultures, enumeration of cultures, etc., those devised by Wright and described by him in various papers in the Lancet during the last four years.

The fact that quite fresh serum has a bactericidal action on some pathogenic bacteria having been established on a firm basis by the work of many observers, the ides suggested itself that here was a means of increasing the virulence of these whenever that should be desirable, by bringing into play the operation of the principle of "the survival of the fittest." For, a given volume of fresh serum having the power of killing off all the bacteria in a given volume of a bacterial emulsion of known strength, and failing to kill them all off in the same volume of an emulsion of greater strength, presumably the survivors would be the ones possessed of the greatest powers of resistance, the slain being those possessed of the least; and by allowing the survivors "the fittest" to propagate their species in serum, and so in each generation eliminating as before the weakly and unfit, one ought ultimately to arrive at a generation of increasedly healthy vigorous and resistant bacteria of the particular species subjected to this selective process, that is, a generation of increased virulence.

With this object in view the following series of experiments were undertaken.

On April 22nd, 19or, sloped iagar [tubes (these throughout the series were all of the same length and diameter and contained identical quantities of agar sloped at the same angle) were inoculated as "mass" cultures of anthrax from an existing laboratory culture by making an emulsion of this with I per cent. peptone broth, allowing this emulsion to flow over the whole agar surface of each of the four sloped agar tubes, which were then inverted to allow as much emulsion to drain off as would, and these four tubes were then incubated at $37^{\circ} \mathrm{C}$. for twenty-four hours, at the end of which, in order to get an index of its virulence, the resulting growths were all combined into one emulsion with ro c.cm. of $x$ per cent. peptone broth, which was injected into a 'guinea-pig of weight 320 grams, and at the same time another/sloped agar tube was inoculated and placed in the incubator at $37^{\circ} \mathrm{C}$., to be used as the basis of the succeeding experiments.

The guinea-pig just mentioned survived the administration of the four anthrax tubes and was alive some weeks after. Meanwhile the tube inoculated and incubated had its resulting plentiful growth of anthrax made into a thick emulsion with broth on April 25th, from which

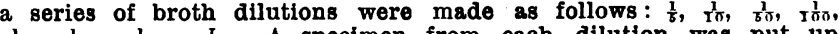
$\frac{1}{1}$, $\frac{1}{2}$, in a capillary tube (calibre of about $\frac{1}{3} \mathrm{~mm}$.) with an equal volume of freshly-drawn serum obtained from myself, the two fluids intimately mixed; the tube then sealed off in a Bunsen flame, indexed, and the whole series incubated at $37^{\circ} \mathrm{C}$. for twenty-four hours. Next day the contents of these capillary tubes were expelled on divisions of an agar surface contained in a Petri dish, which was in its turn incubated at $37^{\circ} \mathrm{C}$. for twenty-four hours, with the result that in each area growth had taken place.

Second Generation.-Taking the growth from the weakest dilution го as there being fewer bacilli in this they would have been most acted on by the bloodsserum-it was emulsified in broth, the same dilutions made and the series repeated exactly as before, with the result that again there was growth from each dilution.

Third Generation.-Taking, as before, the growth from the weakest dilu tion I Th bacilli should be killed off by the bactericidal action of the serum, and then to make use of the growth from the next less weakest dilution for the production of the succeeding generation. This time it was found that theng again there was growth from each dilution, that from the dilution was much more limited than from the others.

Fourth Generation.- Repeated as for third generation, using growth from Tho dilution. This time it was found there was no growth from the rothers.

Fifth Generation-Repeated as before, using the lgrowth from dilution of fourth generation, and each dilution from $\frac{1}{10}$ to ros growth. In order to test if any progress was being made, the growth from the one sloped agar tube, which, after twenty-four hours at $37^{\circ} \mathrm{C}$., wa suspended in 5 c.cm. of broth, and on May roth injected into a healthy pring had risen to $39.8^{\circ} \mathrm{C}$., on the 12 th $40^{\circ} \mathrm{C}$., and on the $3_{3}$ th it was found dead in its cage. A necropsy was made with the customary precautions, and B. whence, as the first guinea-pig had survived the administration of four exactly similar tubes of the orieinal culture, it was deduced that some progress had been made in the direction of increasing virulence.

The growth from the dilution of the same generation was used The gring fown until the fourteenth generation had been reached. Concurrently with these generations passaged through fresh blood serum a series of generations of anthrax passed throug the same original growth, but on agar alone, had been lopt so that on 3 oth one had for puposes of comparing (a) the fourteenth tericidal action of fresh blood stream, and was, on the hypothesis genoration of a series which had grown on agar alone, and was presumably mild relative to the other; both $(a)$ and $(b)$ having originated from the same original growth; and all that remained to be done was to test them comparatively.

In the case of anthrax, owing to the adhesion of the bacilli to each of ondertaken in the case of typhoid bacilli is possible, and the best way of obtaining equivalent doses for administration seemed to be that of preparing twenty-four hours' mass cultivation in exactly similar sloped agar 